DE

M E D I C I N A

T R O P I C A L

$\mathrm{DE}$

SÃO PAULO

JOURNAL OF THE SÃO PAULO INSTITUTE OF TROPICAL MEDICINE

${ }^{1}$ Fiocruz Amazônia, Instituto Leônidas e Maria Deane, Laboratório de Diagnóstico e Controle de Doenças Infecciosas na Amazônia, Amazonas, Manaus, Brazil

${ }^{2}$ Fiocruz, Instituto Oswaldo Cruz, Programa de Pós-Graduação Stricto Sensu em Biologia Celular e Molecular, Rio de Janeiro, Rio de Janeiro, Brazil

${ }^{3}$ Centro Universitário Fametro, Manaus, Amazonas, Brazil

${ }^{4}$ Universidade Federal do Amazonas, Instituto de Ciências Biológicas, Programa de Pós-Graduação em Imunologia Básica e Aplicada, Manaus, Amazonas, Brazil

${ }^{5}$ Fundação Hospitalar de Hematologia e Hemoterapia do Amazonas, Manaus, Amazonas, Brazil

${ }^{6}$ Universidade Federal do Amazonas, Instituto de Ciências Biológicas, Programa de Pós-Graduação em Biotecnologia, Manaus, Amazonas, Brazil

Correspondence to: Luís André Morais Mariúba

Fiocruz Amazônia, Instituto Leonidas e Maria Deane, Rua Terezina, 476, CEP 69057-070, Manaus, AM, Brazil

E-mail: andre.mariuba@gmail.com, lamariuba@outlook.com

Received: 20 May 2020

Accepted: 20 October 2020

\section{Circumsporozoite Surface Protein-based malaria vaccines: a review}

\author{
Maria Edilene Martins de Almeida ${ }^{1,2}$, Maria Gabriella Santos de \\ Vasconcelos $^{1,3}$, Andréa Monteiro Tarragô ${ }^{\text {4,5 }}$, Luís André Morais Mariúba 1,2,4,6
}

\section{ABSTRACT}

Malaria represents a serious public health problem, presenting with high rates of incidence, morbidity and mortality in tropical and subtropical regions of the world. According to the World Health Organization, in 2018 there were 228 million cases and 405 thousand deaths caused by this disease in the world, affecting mainly children and pregnant women in Africa. Despite the programs carried out to control this disease, drug resistance and invertebrate vector resistance to insecticides have generated difficulties. An efficient vaccine against malaria would be a strategy with a high impact on the eradication and control of this disease. Researches aimed at developing vaccines have focused on antigens of high importance for the survival of the parasite such as the Circumsporozoite Surface Protein, involved in the pre-erythrocytic cycle during parasites invasion in hepatocytes. Currently, RTS'S is the most promising vaccine for malaria and was constructed using CSP; its performance was evaluated using two types of adjuvants: AS01 and AS02. The purpose of this review was to provide a bibliographic survey of historical researches that led to the development of RTS'S and its performance analysis over the decade. The search for new adjuvants to be associated with this antigen seems to be a way to obtain higher percentages of protection for a future malaria vaccine.

Keywords: Malaria. Vaccine. Adjuvant. Circumsporozoite Surface Protein.

\section{INTRODUCTION}

Malaria represents a serious public health problem; it has high rates of incidence, morbidity and mortality in tropical and subtropical regions of the world. Despite efforts made with measures to control and prevent the disease, in 2018, 228 million cases and 405 thousand deaths were registered worldwide. Plasmodium falciparum was responsible for $99.7 \%$ of registered cases of malaria in African regions, as well as most cases in Southeast Asia (50\%), Eastern Mediterranean (71\%) and Western Pacific $(65 \%)^{1}$. Thus, research has been carried out aiming to obtain an accessible, safe and efficient vaccine against the parasite, for a long-term control and possible eradication of malaria.

The RTS'S/ AS01 vaccine was designed for the pre-erythrocytic stage of $P$. falciparum, to activate the immune system against the parasite, as soon as it enters the human bloodstream, preventing the sporozoites from invading human hepatocytes, eliminating infected hepatocytes or impairing the development of Plasmodium, making it impossible for the parasite to invade red blood cells ${ }^{2}$. This vaccine uses part of the central repeat domain and the C-terminal region of the P. falciparum Circumsporozoite Surface Protein (CSP), also presenting 
T-cell epitopes coupled to the hepatitis B HBsAg surface antigen $^{3,4}$.

Currently, the RTS'S/ AS01 vaccine is known commercially as Mosquirix ${ }^{\mathrm{TM}}$, and is produced by GlaxoSmithKline Biologicals (GSK SA, United Kingdom). This vaccine received a favorable decision from the European Medicine Agency (EMA) in July 2015, with approval of its quality, safety and effectiveness considering benefits and risks ${ }^{5}$. It is important to highlight that RTS'S/ AS01 has also received authorization from the World Health Organization (WHO) to carry out pilot tests in the Malaria Vaccine Implementation Program (MVIP) and is being currently tested in children and pregnant women living in endemic African countries, mainly in Sub-Saharan Africa ${ }^{6}$.

In the three African countries (Ghana, Kenya and Malawi) selected for the pilot tests, the vaccine follows the Expanded Program on Immunization (EPI) distribution regime, which consists of four doses. In Malawi, the vaccine is offered for the first time at 5 months of age, while in Ghana and Kenya, the vaccine is offered at 6 months of age, with the first three doses delivered at approximately monthly intervals and the fourth dose close to 2 years of age ${ }^{7}$.

The malaria vaccine is also supported by different WHO partner institutions, that are working to validate its effectiveness and distribution in pilot immunization programs. PATH's Malaria Vaccine Initiative (MVI) provides technical support and project management for the program, and Gavi - The Vaccine Alliance, is an international organization that brings together the public and private sectors with the common goal of creating equal access to new and underused vaccines for children living in the poorest world countries 6 .

This vaccine was $50 \%$ effective in children of up to one year, while in adults, for six months ${ }^{8}$. However, there is still a need to enhance the RTS'S vaccine to obtain better immune responses to recombinant antigens, so that different formulations have been developed and tested, using new adjuvants that are linked to immunomodulators, adapted to the antigen and the population that will receive it. Adjuvants can activate the entire immune system, enhancing the vaccine's adaptive response ${ }^{9}$.

RTS'S/ AS02 vaccine is still in phase IIb clinical trial and uses the adjuvant AS02. The adjuvant AS01 (formulated in liposomes) and AS02 (formulated in oilin-water emulsion), consist of the following compounds: Monophosphoryl lipid A (MPL), which functions as an agonist of the TLR4 receptor, activating T-helper lymphocytes 1 (Th1) to produce INF- $\gamma$, IL-2 and TNF- $\alpha$ cytokines, that are related to phagocytosis-mediated defense against intracellular infectious agents; and Quillaja saponaria Molina, fraction 21 (QS21), that initiates the activation of dendritic cells to induce immune responses mediated by $\mathrm{T}$ cells ${ }^{9,10}$.

Thus, this article aimed to present a brief history of the findings that led to the development of the malaria vaccine using the CSP protein as a target antigen, for the production of the RTS'S/ AS01 vaccine and what are the latest findings on its effectiveness.

\section{Brief historical survey of malaria vaccine development}

Since the 1920s, adjuvants have been developed and perfected to increase the immune response against specific antigens in order to improve the efficiency of vaccines in general. Ramon et al. ${ }^{11}$, in 1926, demonstrated that adding substances such as agar, metal salts, oil, lecithin or saponin to the vaccine would artificially increase the levels of tetanus and diphtheria antitoxins.

Later in 1931, Glenny et al. ${ }^{12}$ tested rabbits and guinea pigs with the bacterium Corynebacterium diphtheriae, which causes diphtheria. In this study, aluminum hydroxide (Alum) was used for the first time as an adjuvant, and later it would be used in more modern formulations of a vaccine against Malaria. However, for certain applications, alum salts proved to be of limited use due to their inability to stimulate cell-mediated immunity with greater specificity, local reactions and production of IgE antibodies ${ }^{13}$. For this reason, it was necessary to develop new and more effective adjuvants for malaria vaccines.

In 1945, Jules Freud et al. ${ }^{14}$ carried out the first malaria vaccine tests. These products were used to immunize ducks injected with inactive $P$. lophurae in association with formalin (a substance similar to lanolin), paraffin oil and Koch's bacillus. This organization became known as Freund's Adjuvant. Immunized ducks had a good protection against $P$. lophurae infection, but the vaccine showed some adjuvant-induced reactions. Still, in that same year, the group studied, but this time using $P$. knowlesi to inoculate Rhesus monkeys. The results obtained were similar to the previous ones ${ }^{14}$.

In 1967, mice were immunized with $P$. berghei sporozoites attenuated by X-radiation through studies carried out at the New York University by Ruth Nussenzweig et al. ${ }^{15}$. The results showed that when these animals were challenged with non-irradiated sporozoites, there was a decreased infectivity of sporozoites to infect hepatocytes. Later, the same result was observed in non-human primates ${ }^{16}$.

Based on the Nussenzweig's experiment, in 1971, at the Walter Reed Military Medical Center, Clyde et al. ${ }^{17}$ carried out the first tests on humans. The study was conducted in six adult male volunteers through mosquito bites with X-radiation attenuated sporozoites (P. falciparum, P. vivax, 
and both species), and it was observed that the volunteers had low-level parasitemia. Then, a new study was proposed with these same volunteers, using the same methodology, whose number and degree of infection was sufficient to induce the disease in all volunteers who had participated in previous tests conducted by Clyde et al. ${ }^{17}$. As expected, all unvaccinated volunteers developed malaria. However, only one of the six immunized volunteers obtained protection against the disease.

The advent of the monoclonal antibody technology has allowed advances in the identification of possible vaccine candidates for the parasite in the pre-erythrocytic stage, through the presentation of one or more antigens of this protozoan, instead of using the entire parasite ${ }^{18,19}$ (Figure 1). These advances have led to the principle of discarding vaccines based on attenuated sporozoites in humans. The concentration of these studies, directed at the protective response induced using attenuated sporozoites, led to the identification of the circumsporozoite protein (CSP).

\section{CSP antigen: a vaccine candidate}

Among the main vaccine antigens, CSP has stood out, both for being present in all species of Plasmodium, and for the large amount present on the surface of the parasite, playing a fundamental role in the invasion of parasites into hepatocytes ${ }^{20}$. It has high immunogenicity in human hosts, primates, rodents, and has similar structural and immunological characteristics. For these reasons, CSP was the first protein to be used in different malaria vaccines and remains the main candidate protein for the development of pre-erythrocytic vaccines for the disease ${ }^{21}$.

CSP has approximately 420 amino acid residues, its precursor has $50-70 \mathrm{kDa}$ and, later, it has been cleaved into surface proteins with sizes varying between $40-60 \mathrm{kDa}^{22}$. In $P$. falciparum, the gene encoding the CSP protein is a single copy gene subdivided into three regions (Figure 2).

1) N-terminal region (RI), binds to heparan sulfate proteoglycans, which include the binding domain of several human HLA epitopes ${ }^{23}$. Rathore et $a l .{ }^{20}$ observed that by deleting six amino acids from this region of the P. falciparum CSP, parasites were unable to bind to hepatocytes, preventing the sporozoite invasion process. Besides, it has also been shown that monoclonal antibodies capable of preventing the proteolytic cleavage in the $\mathrm{N}$-terminal region of $P$. falciparum CSP, has the same effect. These data highlight the importance of this region in the construction of a pre-erythrocytic vaccine against Plasmodium.

\section{RTS'S vaccine Phase II - clinical trials in adults and children, with the RTS'S vaccine. \\ Improvement of vaccine \\ formulations using AS01 and AS02 adjuvants.} characterization of the CSP on the surface of the parasite $P$. falciparum

\author{
Phase IV - Evaluation \\ of vaccine safety and \\ efficacy in populations \\ living in endemic \\ areas in Africa
}

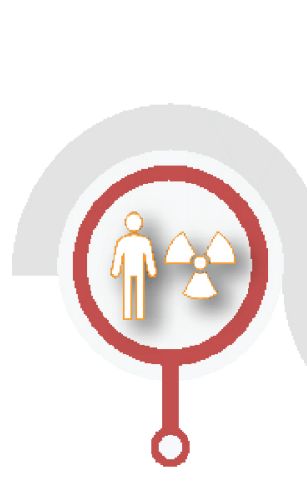

1960 to 1975

\section{9 to 1983}

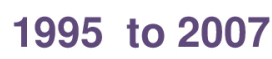

\section{5 to 2019}

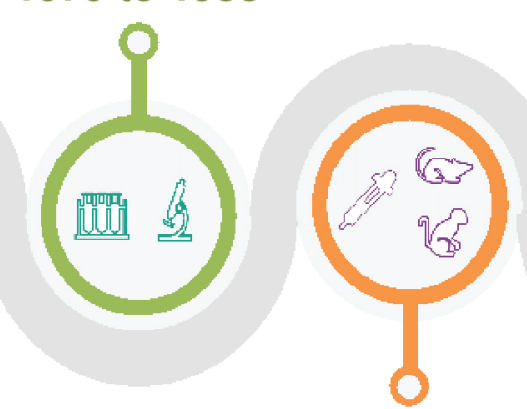

1984 to 1987

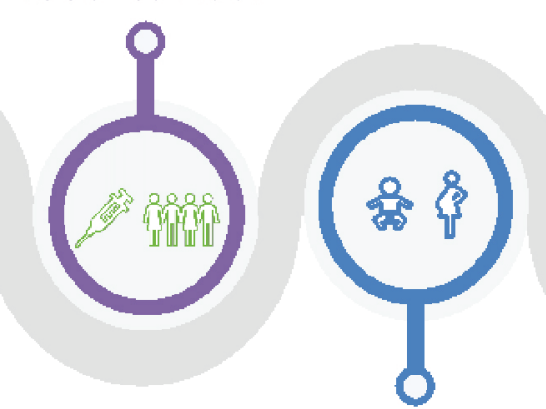

\section{9 to 2015}

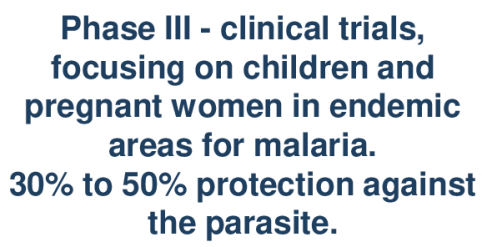

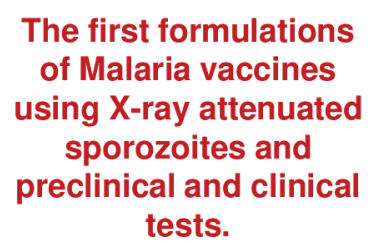

\author{
Coupling of the CSP \\ antigen to the $\mathrm{HbsAg}$. \\ Phase I - Vaccine \\ immunogenicity test in \\ vitro and pre-clinical \\ trials.
}

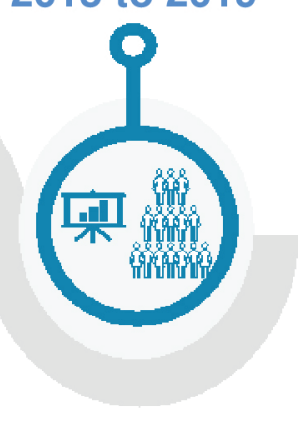

.

Figure 1 - Schedule on the malaria vaccine development for approximately 50 years until 2019. 


\section{P. falciparum CSP}
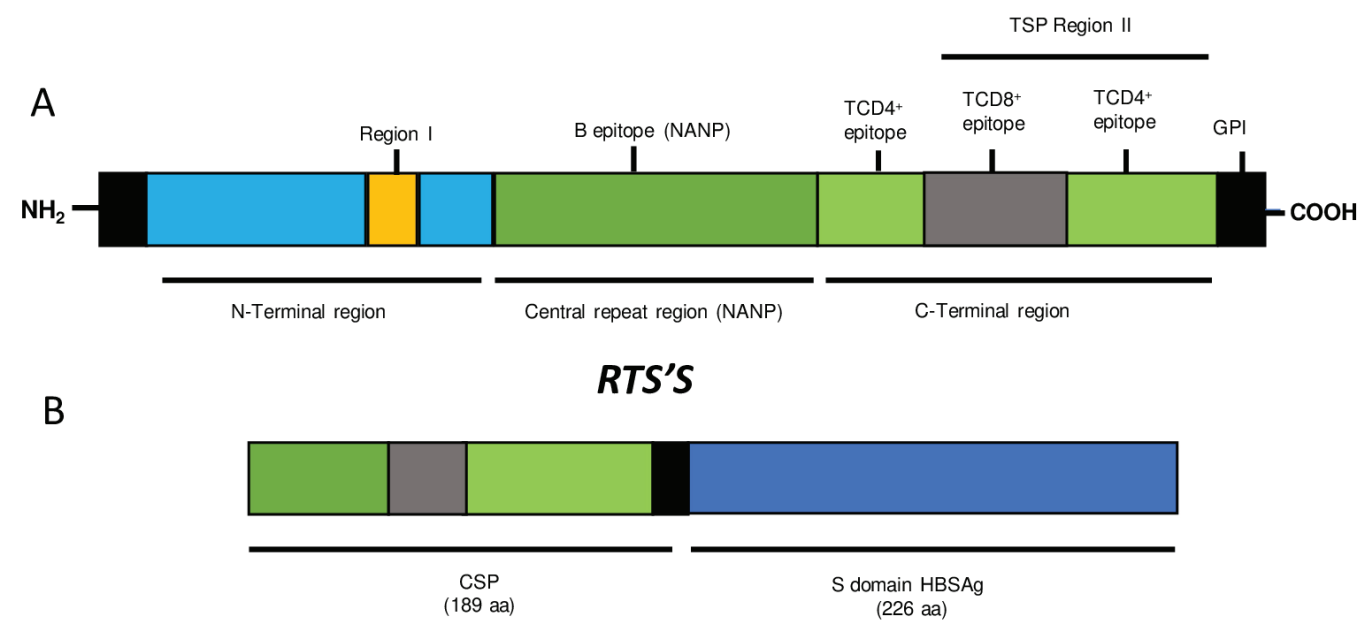

Figure 2- Structure of $P$. falciparum CSP. Approximately 420 amino acid residues. A: $P$. falciparum CSP is subdivided into three regions: CSP comprises an N-terminal region containing a signal peptide sequence and Region I; a Central region containing fouraminoacid (NANP) repeats and a B epitope; and a C-terminal region containing Region II [a thrombospondin (TSP)-like domain] and a canonical glycosylphosphatidylinositol (GPI) anchor additional sequence in addition to three T cell epitopes. The first epitope is CD4+ T cell (Th2R), which is located before the TSR, the second epitope is the CD8+ T cell (Th3R) within the TSR region and a "promiscuous" CD4 T cell epitope (CS.T3). B: The CSP region included in the RTS'S vaccine comprises the last 18 NANP repeats and C-terminus exclusive of the GPI anchor additional sequence. It contains 189 amino acids from CSP and 226 aa from Hepatitis B virus surface antigen (HBsAg) in RTS'S which are genetically fused to the truncated CSP and serve as protein carriers. The CSP fragment in RTS'S contains three known T-cell epitopes: a highly variable CD4+ T-cell epitope before the TSP-like domain (TH2R), a highly variable CD8+ T-cell epitope within the TSP-like domain (TH3R), and a conserved "universal" CD4 + T cell epitope (CS.T3) at the C-terminus.

2) Region of repetition, located in the center of the protein, presenting with amino acids repetitions (NANP), alternated by regions that are predominantly not repeated. In P. falciparum, this region consists of the tetrapeptide NANP and three variants NVDP ${ }^{19}$. This domain contains the majority of immunodominant B-cell epitopes for the production of specific antibodies to P. falciparum ${ }^{24}$ playing a key role in the parasites growth since experiments carried out removing this region led to the development of abnormal sporozoites with degeneration of oocysts and consequent death of these parasites.

3) C-terminal region: this contains a region of thrombospondin (TSR), also known as Region II (R II), anchoring glycosylphosphatidylinositol (GPI). A TSR is very similar to what is present in the human host, acting as a camouflage, delaying or giving time for the parasite to reproduce and respond to the host immune system, in addition to having the benefit of contributing to the proper development of sporozoites in the mosquito's salivary gland $^{25}$. The C-terminal region of the protein contains three T-cell epitopes: the first epitope is CD4+ T cell (Th2R), which is located before TSR, the second epitope is CD8+ T cell (Th3R) within the region TSR and a "promiscuous" CD4 T-cell epitope (CS.T3). This method is conserved among all parasites ${ }^{26}$.

\section{RTS'S vaccine clinical trials.}

A vaccine candidate, after its discovery, goes through elaborate stages of development until its approval as a commercial product. Thus, regulatory agencies around the world share this clinical process (in vitro tests on live animals) and clinical trials on humans. Clinical trials evaluate the safety and effectiveness of a possible candidate through four different stages: I, II, III and IV ${ }^{27}$.

Phase I trials evaluate the first dose of a vaccine administered to humans, the main objective of which is to test the safety, dosage, route of administration and effectiveness of the immune response in a small number of volunteers from an endemic and/ or non-endemic region for the disease. Phase II trials analyze a much larger population in its endemic setting and in multicentric locations. In this case, it evaluates whether the vaccine will be immunogenic and protective against what it was designed for. Phase II trials can be further divided into 1) Phase IIa, which provides preliminary data, through human challenge studies on vaccine efficiency in individuals who have never had the disease or who had it but are healthy in the first time point; 2) Phase IIb, the safety and efficiency of the vaccine is tested, compared to a placebo or standard vaccine used previously. Phase III trials, on the other hand, assess the incidence of the disease in vaccinated and unvaccinated 
volunteers, attesting to the final effect of the vaccine formulation. This phase III can also be divided into 1) phase IIIa, in which the results obtained are presented to the Regulatory Agency for the later registration and approval of the product for the market; 2) Phase IIIb, changes are made when necessary in the vaccine for approval and registration with the regulatory agency. While, in phase IV, post-marketing surveillance studies (PMS) begin, which are designed to continue monitoring the product in this way, ensuring safety and effectiveness in the target population ${ }^{27}$.

In 1985, the Walter Reed Army Research Institute (WRAIR) in partnership with the company GlaxoSmithKline Biologicals (GSK) initiated experiments for a recombinant vaccine using the circumsporozoite protein (CSP), a tetrapeptide repeat sequence found and expressed in the bacterium Escherichia coli and inoculated into mice. These produced high concentrations of antibodies against CSP, when evaluated in vitro and these antibodies were able to block the invasion of sporozoites in human hepatocyte cells, highlighting the importance of CSP as a vaccine candidate ${ }^{21}$.

In 1987, new formulations of the malaria vaccine were developed, as well as other adjuvants that were tested to improve their performance. A vaccine containing the recombinant protein from the tetrapeptide from the CSP repeat region was developed and tested in mice using aluminum hydroxide (Alum). Two groups were evaluated: (1) CSP + Alum group and (2) Control group (Without Alum). The CSP + Alum group showed high concentrations of antibodies when compared to the control group (without Alum). This new vaccine formulation using an adjuvant, generated antibodies that inhibited the invasion of sporozoites to human hepatocytes in vitro, validating the CSP antigen to generate protection against the human malaria parasite and the importance of using adjuvant in the formulation as a way to perfect the vaccine ${ }^{18}$.

Human clinical trials started in 1987, using synthetic peptides containing $P$. falciparum-CSP immunodominant epitopes conjugated to tetanus toxoid (TT) and using Alum as an adjuvant; 35 healthy adults were administered intramuscularly with three doses in monthly intervals. The individuals' sera showed reactivity of $53 \%$ and $71 \%$ against NANP as the concentration of these peptides increased when 100 and $160 \mathrm{mg}$ were used, respectively. The vaccine was shown to be safe, with no adverse reactions, only pain at the inoculum site. After immunizations, three individuals with higher antibody titers and four control volunteers were challenged with $P$. falciparum sporozoites inoculated by infected mosquito bites. After the challenge, all controls showed the disease, while $2 / 3$ of the vaccines did not show parasitemia until the eleventh day; $1 / 3$ of those vaccinated did not show parasitemia or symptoms of the disease during the other 29 days of the observed period. This vaccine prototype was considered safe and stimulated the production of antibodies in these individuals, but with short-term protection, requiring new, more efficient formulations to help controlling malaria ${ }^{28}$.

To increase and prolong the effectiveness of the vaccine application, in 1988, Rutgers et al, began the tests using a central repeat region of the P. falciparum CSP protein, fused after exposure to the hepatitis B surface antigen (HBsAg) as a carrier matrix for major B cell epitopes ${ }^{29}$.

To improve the application of the vaccine, important $\mathrm{T}$ and $\mathrm{B}$ cells epitopes in the C-terminal region of the CSP protein were included. In 1989, Nardin et al. ${ }^{19}$ carried out an experiment to clone $\mathrm{T}$ cells taken from a human volunteer, who was immunized with the old native CSP and the recombinant CSP protein. After the challenge with sporozoites, immunological responses were highly specific for a repeat region of the CSP, the "NANPNVDPNANP" sequence, highlighting the importance of this region in the composition of a malaria vaccine.

In 1991, the CSP formulation was redesigned to contain 19 NANP repeats and the C-terminal region (amino acids 210-398) of the CSP antigen, along with 226 amino acids of the hepatitis B surface antigen (HbsAg). The current malaria vaccine formulation is known as RTS'S produced and adsorbed with alum adjuvant ( RTS'S/ Alum) ${ }^{30}$.

In 1995, one of the first phase I studies in human immunization was carried out using the new formulation of the RTS'S vaccine. The test was carried out on 20 volunteers without malaria, 10 of whom received RTS'S/ Alum and 10 received the RTS'S/Alum/MPL vaccine. Both formulations proved to be safe and immunogenic, as they induced the production of high levels of antibodies in vaccinated individuals. Subsequently, volunteers were challenged with sporozoites. Post-challenge, all individuals in the RTS/ Alum group and 75\% in the RTS/Alum/MPL group developed mild malaria. The RTS/ Alum/MPL group showed higher concentrations of antibodies against CSP on the day of the challenge compared to individuals who received only RTS/ Alum, but these antibodies were not sufficient to generate a vaccine protection ${ }^{24}$.

In 1997, new tests were reinitiated with the RTS'S vaccine, only this time the CSP protein in addition to fused to HBsAg was expressed together with unfused HBsAg. This study aimed at improving the safety and efficacy of the vaccine using more potent adjuvants, in an attempt to increase the immune response of immunized individuals. For this, a clinical trial was carried out using three vaccine formulations containing RTS'S. In total, 46 individuals who had not been exposed to malaria, aged 18 to 45 years old were evaluated and received $1 \mathrm{~mL}$ of "Vaccine 1" (RTS'S + 
SBAS4- Alum and MPL); $0.5 \mathrm{~mL}$ of "Vaccine 2" (RTS'S + SBAS3- Oil-in-water emulsion); and $0.5 \mathrm{~mL}$ of "Vaccine 3" (SBAS2-MPL and QS21). Only 22 immunized individuals and six non-immunized controls were challenged with mosquito bites contaminated with P. falciparum. However, of the seven individuals who took the three doses of "Vaccine 3", six volunteers did not develop the disease. The researchers concluded that a recombinant vaccine based on the fusion of CSP with the Hepatitis B antigen (HBsAg) in addition to a potent adjuvant could protect adult individuals who have never had contact with malaria ${ }^{3}$.

From the results obtained in previous studies, Stoute et al.$^{31}$ followed-up the volunteers, this time to determine the longterm effectiveness of the immune response in individuals who had been immunized with RTS'S. It was found that the levels of antibodies were decreasing six months after the last dose of the vaccine. Seven volunteers from the previous study agreed to participate in a new challenge six months later. In both challenges, all controls developed parasitemia and only one volunteer who received "vaccine 1" was protected in both challenges. In all volunteers who underwent the challenge and became infected, the average pre-patient period was 10.8 days for the control group and 13.2 days for the vaccinated one, and this increment was statistically significant. The research observed the proliferation of T cells and the production of interferon- $\gamma$, highlighting that the response to RTS'S was superior when compared to responses to HbsAg. However, they observed that there was no correlation between the immune response and protection against the disease.

\section{RTS'S/AS02}

In 2001, another research was developed to optimize the dose of "Vaccine 3" using the adjuvant AS02 (RTS'S/AS02A). For this, a controlled phase I and II study was carried out evaluating the number of standard doses of the vaccine in 41 volunteers for vaccination. The vaccine was shown to be safe and immunogenic in all participants. After the immunization stage, 41 vaccinated and 23 individuals without malaria (controls) were submitted to the sporozoite challenge. After the application of the three doses of the vaccine, an overall efficacy of $41 \%$ was observed, starting from the second dose, demonstrating a partial protection against the challenge with $P$. falciparum sporozoites $^{32}$.

In 2001, a randomized clinical trial was conducted to test the effectiveness of the RTS'S/ AS02 vaccine on 306 semiimmune men aged 18 to 45 years old in the Gambia. One of the groups received three doses of the RTS'S/AS02 vaccine (test group) and the other three doses of rabies vaccine (control group). The control group, as expected, developed malaria earlier than the test group, in which a strong humoral response against CSP was shown. In the first nine weeks, the effectiveness of the RTS'S/AS02 vaccine was 71\%. However, it declined to $0 \%$ after six weeks. Finally, 158 men received a fourth dose of the vaccine the following year (2003) and were followed-up again for nine weeks. After the fourth dose, the vaccine was $47 \%$ effective $^{33}$.

Other studies carried out with 214 Mozambican children using the RTS'S/ AS02 vaccine. Good results have been observed against the first malaria infection, and the immunity lasted up to six months after the third dose of the vaccine ${ }^{34}$. Similar results were found in this same population, in which the efficacy of the vaccine against clinical malaria was 33\%, showing safety, immunogenicity over 14 months after the beginning of the study, however a decrease in anti-CSP antibodies was observed during the vaccination period ${ }^{35}$.

Recent research has shown that antibodies induced by the RTS'S/AS02 vaccine can interact with the complement system. The study was conducted from a randomized phase IIb clinical trial with the RTS'S/AS02 vaccine in children, in Mozambique. Vaccination with RTS'S/AS02 induced anti-CSP antibodies mainly of the IgG1 subclass. . It was observed that these antibodies induced by the RTS'S/ AS02 vaccine were able to fix and activate the complement system, through the recruitment of C1q. This discovery implies the elimination of the pathogen through the joint action of antibodies with the complement system. Besides, a decrease in antibodies (IgG and $\operatorname{IgM}$ ) for complement fixation was observed a few months after vaccination, making it difficult to obtain immunological memory ${ }^{36}$.

\section{RTS'S/AS01}

Pre-clinical studies have been carried out to identify new adjuvant formulations for use with RTS'S to achieve a significant and prolonged specific immunity duration.

In 2006, a study was carried out to distinguish the best formulation of adjuvants for the RTS'S vaccine through immunizations in primates (Rhesus macaques) using RTS'S with four formulations of adjuvants: RTS'S/AS01B; RTS'S/AS02A (Standard); RTS'S/ AS05 and RTS'S/AS06. It was observed that among the adjuvants used, AS01B was the one with the highest protection index between $14^{\text {th }}$ and $34^{\text {th }}$ weeks, surpassing the AS02A standard. Thus, they observed that RTS'S/ AS01B was the most effective and superior immunogenic formulation for cellular responses, compared to RTS'S/ AS02A (standard) ${ }^{37}$. From March 2009 to January 2011 a phase 3 study was carried out with infants aged five to 17 months and infants aged six to 12 weeks in eight countries, in Sub-Saharan Africa. One year after the third dose of RTS'S/ AS01, the effectiveness of protection against the parasite was observed in $55.8 \%$ of 
infants aged five to 17 months and $31.3 \%$ of infants aged six to 12 weeks $^{38}$. The participants were followed-up for four years. One year after the $3^{\text {rd }}$ dose, it was found that the vaccine was able to protect this population. However, after this period, the antibody serum levels against CSP decreased in both populations ${ }^{8}$.

In 2019, the most recent phase 3 study with the RTS'S/ AS01 vaccine was carried out with a population of 109 infants aged six to 12 weeks and 86 infants aged 5 to 17 months. Data from this clinical trial found that vaccination with RTS'S/ AS01 generated antibodies predominantly of the subclasses $\operatorname{IgG} 1$ or IgG3, with lower levels of IgG2 and IgG4. There were associations with the protection of $\mathrm{IgG} 3$ antibodies to selected antigens, followed by the IgG1 subclass. The research has also highlighted the importance of these antibodies for improving the RTS'S/AS01 vaccine ${ }^{39}$.

The RTS'S vaccine during phase 3 trials showed adverse reactions that were considered tolerable and similar to those of other childhood vaccines. Reported side effects were pain and swelling at the injection site and fever ${ }^{6}$.

During this phase there were more reports of meningitis cases in children who received the vaccine, but no association was proven. Thus, EMA concluded that there is not enough information to classify the finding as a potential risk, reasoning that it was probably an occasional finding, but should be monitored during the introduction of the vaccine ${ }^{5,7}$.

Despite reports of adverse effects, reduced duration and low efficacy, RTS'S is the first malaria vaccine that has proven to be effective, as it contributes to the reduction of severe malaria rates and hospitalizations in children aged five to 17 months, which is the most affected group by this disease ${ }^{38}$. Among the three communities in which the vaccine is being implemented (Malawi, Kenya, and Ghana), Malawi welcomed the malaria vaccine. Therefore, the distribution of RTS'S malaria vaccine, even with partial protection, is believed to lead to a significant impact on the health of African children ${ }^{6,7}$.

Nowadays, the malaria vaccine is in phase 4 clinical trial expected to be completed in 2023. At this stage, additional information on the vaccine's effectiveness and side effects associated with the routine use will be gathered. In 2019, the MVIP, WHO and Gavi - The Vaccine Alliance, committed to additional funding to complete the pilot program by $2023^{6}$.

\section{CONCLUSION AND PERSPECTIVES}

Therefore, both the RTS'S vaccine using adjuvant AS01 and $\mathrm{ASO} 2$ have points to be improved. Although the reason for this partial generation of protection is not yet clear, the research previously cited indicates that the polymorphism in the central and C-terminal regions, as well as the particularity of each adjuvant and the age of the vaccines are factors that influence their effectiveness.

Other obstacles still have to be overcome for the development of an effective vaccine against malaria, such as the genetic diversity present in the Central repeating regions and the C-terminal of $P$ falciparum CSP that makes up the RTS'S/ AS01 vaccine. These polymorphic regions make it difficult to obtain immunological memory, a limitation that has been observed in clinical studies in different areas endemic for malaria ${ }^{40}$.

The C-terminal region of the protein has polymorphisms concentrated in the Th2R and Th3R regions, while the central repeat region of the CSP antigen, has polymorphisms in the NANP and NVDP repeat sequences. These data were retrieved from different geographic areas, mainly in African, Asian and Ocean continents ${ }^{41}$. These types of studies underscore the importance of incorporating population genetic studies into the future malaria vaccine project, and it is considered revealing to include the assessment of the diversity of Pf CSP C-terminal in the malaria vaccine implementation program and to further improve the understanding of how genetic diversity affects the effectiveness of the RTS'S/ AS01 vaccine ${ }^{42}$.

Currently, through simulations, researchers have been finding other ways to deal with Plasmodium, one of which is to associate the vaccine with anti-malarial drugs. This new alternative has a great potential for impact on endemic regions since the combination of the vaccine-drug combination will decrease the prevalence of resurgence of transmission after the end of vaccination. The joint action of vaccines and medicines reduces the number of rounds and years of intervention to achieve the same impact on public health as chemoprevention, in addition to no age differentiation ${ }^{43}$.

More research is needed to ensure the development of an effective vaccine against the malaria parasite, contributing to a longer-lasting, safe immunological memory for all age groups and a long-term control and possible eradication of malaria. The use of other proteins as immunogenic as CSP can be a new way of experimenting to create a safer formulation of a multivalent vaccine. The importance of analyzing new adjuvants for the composition of these vaccines, such as liposomes and Bacillus subtilis spores which showed promising results for other antigens, but still need further evaluation in animal and human models is also noteworthy.

\section{ACKNOWLEDGMENTS}

We would like to thank the FIOCRUZ support Foundation - Fiotec, the Postgraduate Program in Cellular and Molecular Biology-PGBCM of Instituto Oswaldo Cruz- 
IOC / FIOCRUZ, and Leonidas and Maria Deane Institute - ILMD / Fiocruz Amazonia for funding the scholarship.

\section{CONFLICT OF INTERESTS}

The authors state no conflicts of interest.

\section{REFERENCES}

1. World Health Organization. World malaria report 2019. Geneva: WHO; 2019.

2. Garçon N, Chomez P, Van Mechelen M. GlaxoSmithKline Adjuvant Systems in vaccines: concepts, achievements and perspectives. Expert Rev Vaccines. 2007;6:723-39.

3. Stoute JA, Slaoui M, Heppner DG, Momin P, Kester KE, Desmons $\mathrm{P}$, et al. A preliminary evaluation of a recombinant circumsporozoite protein vaccine against Plasmodium falciparum malaria. RTS,S Malaria Vaccine Evaluation Group. N Engl J Med. 1997;336:86-91

4. Bejon P, Lusingu J, Olotu A, Leach A, Lievens M, Vekemans J, et al. Efficacy of RTS,S/AS01E vaccine against malaria in children 5 to 17 months of age. N Engl J Med. 2008;359:2521 32.

5. European Medicines Agency. First malaria vaccine receives positive scientific opinion from EMA: press release 24/07/2015 [cited 2020 Oct 21]. Available from: http://www.ema.europa. eu/en/news/first-malaria-vaccine-receives-positive-scientificopinion-ema

6. World Health Organization. Q\&A on the malaria vaccine implementation programme (MVIP): march 2020. [cited 2020 Oct 21]. Available from: http://www.who.int/malaria/media/ malaria-vaccine-implementation-qa/en/

7. World Health Organization. First malaria vaccine: a potential new tool for child health and improved malaria control. [cited 2020 Oct 21]. Available from: https://www.who.int/publications/i/ item/who-cds-gmp-2018-05

8. The RTS,S Clinical Trials Partnership. Efficacy and safety of the RTS,S/AS01 malaria vaccine during 18 months after vaccination: a phase 3 randomized, controlled trial in children and young infants at 11 African sites. PLoS Med. 2014;11:e1001685.

9. Didierlaurent AM, Laupèze B, Di Pasquale A, Hergli N, Collignon C, Garçon N. Adjuvant system AS01: helping to overcome the challenges of modern vaccines. Expert Rev Vaccines. 2017; 16:55-63.

10. Newman MJ, Wu JY, Gardner BH, Anderson CA, Kensil $\mathrm{CR}$, Recchia J, et al. Induction of cross-reactive cytotoxic T-lymphocyte responses specific for HIV-1 gp120 using saponin adjuvant (QS-21) supplemented subunit vaccine formulations. Vaccine. 1997;15:1001-7.

11. Ramon G. Procédés pour accroître la production de antitoxines.
Ann Inst Pasteur. 1926;40:1-10.

12. Glenny AT, Buttle GA, Stevens MF. Rate of disappearance of diphtheria toxoid injected into rabbits and guinea-pigs: toxoid precipitated with alum. J Pathol Bacteriol. 1931;34:267-75.

13. Brewer JM. (How) do aluminium adjuvants work/Immunol Lett. 2006;102:10-5.

14. Freund J, Sommer HE, Walter AW. Immunization against malaria: vaccination of ducks with killed parasites incorporated with adjuvants. Science. 1945; 102:200-2.

15. Nussenzweig RS, Vanderberg J, Most H, Orton C. Protective immunity produced by the injection of $\mathrm{x}$-irradiated sporozoites of Plasmodium berghei. Nature. 1967;216:160-2.

16. Gwadz RW, Cochrane AH, Nussenzweig V, Nussenzweig RS. Preliminary studies on vaccination of Rhesus monkeys with irradiated sporozoites of Plasmodium knowlesi and characterization of surface antigens of these parasites. Bull World Health Organ. 1979;57 Suppl 1:165-73.

17. Clyde DF, Most H, McCarthy VC, Vanderberg JP. Immunization of man against sporozite-induced falciparum malaria. Am J Med Sci. 1973; 266:169-77.

18. Hollingdale MR, Ballou WR, Aley SB, Young JF, Pancake $\mathrm{S}$, Miller $\mathrm{LH}$, et al. Plasmodium falciparum: elicitation by peptides and recombinant circumsporozoite proteins of circulating mouse antibodies inhibiting sporozoite invasion of hepatoma cells. Exp Parasitol. 1987;63:345-51.

19. Nardin EH, Herrington DA, Davis J, Levine M, Stuber D, Takacs B, et al. Conserved repetitive epitope recognized by CD4+ clones from a malaria-immunized volunteer. Science. 1989;246:1603-6

20. Rathore D, Sacci JB, de la Vega P, McCutchan TF. Binding and invasion of liver cells by Plasmodium falciparum sporozoites: essential involvement of the amino terminus of circumsporozoite protein. J Biol Chem. 2002;277:7092-8.

21. Young JF, Hockmeye WT, Gross M, Ballou WR, Wirtz RA, Trosper JH, et al. Expression of Plasmodium falciparum circumsporozoite proteins in Escherichia coli for potential use in a human malaria vaccine. Science. 1985;228:958-62.

22. Coppi A, Pinzon-Ortiz C, Hutter C, Sinnis P. The Plasmodium circumsporozoite protein is proteolytically processed during cell invasion. J Exp Med. 2005;201:27-33.

23. Doolan DL, Hoffman SL, Southwood S, Wentworth PA, Sidney J, Chesnut RW, et al. Degenerate cytotoxic T cell epitopes from P. falciparum restricted by multiple HLA-A and HLA-B supertype alleles. Immunity. 1997;7:97-112.

24. Gordon DM, McGovern TW, Krzych U, JC Cohen, Schneider I, LaChance R, et al. Safety, immunogenicity, and efficacy of a recombinantly produced Plasmodium falciparum circumsporozoite protein-hepatitis B surface antigen subunit vaccine. J Infect Dis. 1995;171:1576-85.

25. Doud MB, Koksal AC, Mi LZ, Song G, Lu C, Springer TA. Unexpected fold in the circumsporozoite protein target of 
malaria vaccines. Proc Natl Acad Sci USA. 2012;109:7817-22.

26. Kaslow DC, Biernaux S. RTS'S: toward a first landmark on the malaria vaccine technology roadmap. Vaccine. 2015;33:7425-32.

27. Singh K, Mehta S. The clinical development process for a novel preventive vaccine: an overview. J Postgrad Med. 2016;62:4-11.

28. Herrington DA, Clyde DF, Losonsky G, Cortesia M, Murphy JR, Davis J, et al. Safety and immunogenicity in man of a synthetic peptide malaria vaccine against Plasmodium falciparum sporozoites. Nature. 1987;328:257-9.

29. Rutgers T, Gordon D, Gathoye AM, Hollingdale M, Hockmeyer WT, Rosenberg M, et al. Hepatitis B surface antigen as a carrier matrix for the repetitive epitope of the circumsporozoite protein of Plasmodium falciparum. Bio/Technology. 1988;6:1065-70.

30. Vreden SG, Verhave JP, Oettinger T, Sauerwein RW, Meuwissen JH. Phase I clinical trial of a recombinant malaria vaccine consisting of the circumsporozoite repeat region of Plasmodium falciparum coupled to hepatitis B surface antigen. Am J Trop Med Hyg. 1991;45:533-8.

31. Stoute JA, Kester KE, Krzych U, Wellde BT, Hall T, White K, et al. Long-term efficacy and immune responses following immunization with the RTS,S malaria vaccine. J Infect Dis. 1998;178:1139-44.

32. Kester KE, McKinney DA, Tornieporth N, Ockenhouse CF, Heppner DG, Hall T, et al. Efficacy of recombinant circumsporozoite protein vaccine regimens against experimental Plasmodium falciparum malaria. J Infect Dis 2001;183:640-7.

33. Bojang KA, Milligan PJ, Pinder M, Vigneron L, Alloueche A, Kester KE, et al. Efficacy of RTS,S/AS02 malaria vaccine against Plasmodium falciparum infection in semi-immune adult men in The Gambia: a randomised trial. Lancet. 2001;358:1927-34.

34. Abdulla S, Oberholzer R, Juma O, Kubhoja S, Machera F, Membi $\mathrm{C}$, et al. Safety and immunogenicity of RTS,S/AS02D malaria vaccine in infants. N Engl J Med. 2008;359:2533-44.

35. Aide P, Aponte JJ, Renom M, Nhampossa T, Sacarlal J, Mandomando I, et al. Safety, immunogenicity and duration of protection of the RTS,S/AS02(D) malaria vaccine: one year follow-up of a randomized controlled phase I/IIb trial. PLoS One. 2010;5:e13838.

36. Kurtovic L, Boyle MJ, Opi DH, Kennedy AT, Tham WH, Reiling $\mathrm{L}$, et al. Complement in malaria immunity and vaccines. Immunol Rev. 2020;293:38-56.

37. Stewart VA, Walsh DS, McGrath SM, Kester KE, Cummings JF, Voss G, et al. Cutaneous delayed-type hypersensitivity (DTH) in a multi-formulation comparator trial of the anti-falciparum malaria vaccine candidate RTS,S in Rhesus macaques. Vaccine. 2006;24:6493-502.

38. RTS,S Clinical Trials Partnership. Efficacy and safety of RTS,S/ AS01 malaria vaccine with or without a booster dose in infants and children in Africa: final results of a phase 3, individually randomised, controlled trial. Lancet. 2015;386:31-45.

39. Dobaño C, Ubillos I, Jairoce C, Gyan B, Vidal M, Jiménez A, et al. RTS,S/AS01E immunization increases antibody responses to vaccine-unrelated Plasmodium falciparum antigens associated with protection against clinical malaria in African children: a case-control study. BMC Med. 2019;17:157.

40. Zeeshan M, Alam MT, Vinayak S, Bora H, Tyagi RK, Alam MS, et al. Genetic variation in the Plasmodium falciparum circumsporozoite protein in India and its relevance to RTS,S malaria vaccine. PLoS One. 2012;7:e43430.

41. Lê HG, Kang JM, Moe M, Jun H, Thái TL, Lee J, et al. Genetic polymorphism and natural selection of circumsporozoite surface protein in Plasmodium falciparum field isolates from Myanmar. Malar J. 2018;17:361.

42. Mohamed NS, Ali Albsheer MM, Abdelbagi H, Siddig EE, Mohamed MA, Ahmed AE, et al. Genetic polymorphism of the N-terminal region in circumsporozoite surface protein of Plasmodium falciparum field isolates from Sudan. Malar J. 2019; 18:333.

43. Camponovo F, Ockenhouse CF, Lee C, Penny MA. Mass campaigns combining antimalarial drugs and anti-infective vaccines as seasonal interventions for malaria control, elimination and prevention of resurgence: a modelling study. BMC Infect Dis. 2019;19:920 